\title{
Criterios de evaluación para el bienestar animal en planta de faena de aves
}

\author{
Villamañe, R.'; Trevisi, D.'; Yuño, M. ${ }^{3}$ \\ Veterinario actividad privada ${ }^{1}$; Facultad de Ciencias Agrarias UNMDP ${ }^{2}$; Facultad de Ciencias Veterinarias \\ UNCPBA, Tandil, Buenos Aires, Argentina ${ }^{3}$. E-mail: myunio@vet.unicen.edu.ar
}

\section{Resumen}

Villamañe, R.; Trevisi, D.; Yuño, M.: Criterios de evaluación para el bienestar animal en planta de faena de aves. Rev. Vet. 32: 1, 58-63, 2021. El presente trabajo analiza los aspectos que involucran el bienestar animal (BA) en todo el proceso de faena de aves de producción en la República Argentina. El Servicio Nacional de Sanidad y Calidad Agroalimentaria (SENASA) es la institución responsable de las normas y acciones para implementar el BA en los animales de producción. Estas normas deben incluir las estipuladas por la Organización Multilateral de Comercio para productos de origen animal y son exigidas y auditadas por los mercados para la exportación de productos argentinos. La faena es la etapa donde culmina el ciclo de carne aviar y en la cual se evalúan los efectos de la aplicación del BA en todo el proceso productivo. Las normas de BA vinculadas a la faena incluyen la carga de aves en la granja, transporte, descarga, degüello y procesado hasta la obtención del producto final. La captura y carga de aves en la granja requiere que la tarea se realice en un período breve y personal capacitado que evite lesiones. Los pollos no deben ser sometidos a un período de ayuno mayor a doce horas entre la captura y la insensibilización en planta de faena, lo cual además disminuye la contaminación microbiológica de las carcasas durante el proceso de eviscerado. El agua de bebida no se debe retirar hasta el comienzo de la carga. El tiempo de espera en planta de faena varía según la operatoria y la distancia del viaje, debiendo ser entre 30 minutos y 3 horas. Es importante respetar este tiempo mínimo para que los animales reduzcan el nivel de estrés que se manifiesta por disminución de frecuencia cardíaca y vocalizaciones. Los indicadores de BA en faena son las aves muertas al arribo, las lesiones en carcaza y garras, entre otros.

Palabras clave: bienestar animal, faena, pollos de engorde, calidad.

\begin{abstract}
Villamañe, R.; Trevisi, D.; Yuño, M.: Evaluation criteria for animal welfare in slaughter plants. Rev. Vet. 32: 1, 58-63, 2021. This paper analyzes the aspects that involve animal welfare (AW) in the entire slaughter process of poultry production in the Argentine Republic. The National Service for Agrifood Health and Quality (SENASA) is the institution responsible for the norms and actions to implement the AW in production animals. These standards must include those stipulated by the regulations of World Trade Organization, and are required and audited for the export of Argentine products. The slaughter is the stage where the cycle of poultry meat culminates and in which the effects of the application of AW in the entire production process are evaluated. The AW standards related to slaughter include the loading of birds on the farm, transport, unloading, slaughter and processing until the final product is obtained. Catching and loading birds on the farm requires the task to be done in a short time and trained personnel to avoid injury. Chickens should not be subjected to a fasting period of more than twelve hours between capture and desensitization in the slaughterhouse, which also reduces the microbiological contamination of the carcasses during the evisceration process. Drinking water should not be removed until the start of charging. The waiting time at the slaughter plant varies according to the operation and the distance of the trip, it should be between 30 minutes and 3 hours. It is important to respect this minimum time for the animals to reduce the level of stress that is manifested by decreased heart rate and vocalizations. The indicators of AW in slaughter are dead birds on arrival, carcass and claw injuries, among others.
\end{abstract}

Key words: animal welfare, slaughter, broilers, quality. 


\section{INTRODUCCIÓN}

La Organización Mundial de Sanidad Animal, conocida originalmente como Oficina Internacional de Epizootias (OIE), ha definido que los animales experimentan "bienestar" siempre y cuando estén sanos, bien alimentados, seguros, libres de padecer sensaciones desagradables como dolor, miedo o desasosiego, y cuando son capaces de expresar comportamientos adecuados para su estado físico y mental ${ }^{13}$.

Además, para prevenir o tratar enfermedades, las normas de bienestar animal (BA) incluyen el uso de productos veterinarios y el sacrificio humanitario compasivo, cuando corresponda ${ }^{12,25}$. El concepto de BA, aplicado a esta etapa de la cadena productiva, involucra aspectos reglamentarios, comerciales, productivos, de faena y éticos. Los aspectos reglamentarios están vinculados al comercio nacional e internacional, por lo cual las normas de BA en Argentina incluyen los principios establecidos internacionalmente en el marco de la Organización Multilateral de Comercio para productos de origen animal ${ }^{11}$.

En tal sentido, el Servicio Nacional de Sanidad y Calidad Agroalimentaria (SENASA) ha desarrollado normas y materiales de comunicación, en tanto que sus acciones garantizan a los consumidores los procedimientos de obtención de alimentos inocuos ${ }^{21,22,23}$, $24,25,26,27,28$.

En aspectos productivos, la aplicación de las normas de BA posibilitan la expresión de su potencial de eficiencia. La faena es el último eslabón de la cadena productiva e incluye la carga de las aves en granja, su transporte, arribo a planta de faena, la espera o descanso, descarga, colgado, insensibilizado, degüello, desangrado, escaldado y enfriado, como así también el eviscerado, trozado e inspección sanitaria en frigorífico.

En cada una de estas etapas deben aplicarse las normas de BA. Además, fue importante que los aspectos éticos hayan sido fundamentados desde siglos pasados por estadistas y filósofos tales como Gandhi, Sarmiento, Nietzsche y Schopenhauer -entre otros- quienes contribuyeron a la salud física y mental de los seres humanos ${ }^{6,30}$.

En virtud de la creciente importancia del BA a nivel internacional en todas las especies animales y, en este caso en particular, en la faena de aves, existen publicaciones recientes que contemplan diferentes aspectos de la misma $7,19,31$.

Este trabajo no pretende efectuar una revisión exhaustiva de la temática, como las realizadas en las referencias mencionadas, sino en particular focalizar en normativas e incluir -además- aportes de la propia experiencia de los autores y de la bibliografía de relevancia, relacionada a la aplicación de BA en todo el proceso de faena de aves de producción en las condiciones de la República Argentina.

\section{PREVIO A LA FAENA}

En cada etapa de producción debe minimizarse el estrés, ya que genera aumento de la glicólisis muscular y -como consecuencia- se altera la maduración y calidad final del producto. En caso de producirse glicólisis ante mortem se obtienen carnes oscuras, firmes y secas, mientras que si se produce la glicólisis post mortem se obtienen carnes pálidas, poco firmes y exudativas ${ }^{1}$.

Ambos defectos, no deseables en el producto final, están estrechamente relacionados con el BA y las situaciones de estrés. Para evitar tales efectos, la captura debe realizarse en penumbra ${ }^{26}$. En las granjas, para la captura de las aves, se realizan maniobras rápidas que pueden generar hematomas en muslos, hemorragias y fractura de alas cuando el personal no tiene la capacitación y entrenamiento adecuado ${ }^{17,31}$.

Tal etapa debe realizarse en un tiempo no mayor a 60 minutos y ser controlada por personal de la empre$\mathrm{sa}^{30}$. Al momento de la carga, si hubiese algún animal lesionado o enfermo, se deberá sacrificar mediante eutanasia humanitaria por la técnica de dislocación cervical. Se recomienda que no se tomen las aves por las alas, ni de las patas o cabezas. Ellas deben sujetarse por el cuerpo con firmeza pero con cuidado y colocarlas en las jaulas de transporte.

Estas jaulas tienen características definidas por SENASA ${ }^{24}$ y el número de aves a transportar depende del peso y época del año. De este modo, para un peso de faena de hasta $1,6 \mathrm{~kg}$ se incluyen 12 pollos/jaula en invierno $\left(225,62 \mathrm{~cm}^{2} / \mathrm{kg}\right)$ y 10 pollos/jaula en verano $\left(270,75 \mathrm{~cm}^{2} / \mathrm{kg}\right)$; para un peso de faena hasta $3 \mathrm{~kg}$ se incluyen 8 pollos/jaula en invierno $\left(180,5 \mathrm{~cm}^{2} / \mathrm{kg}\right)$ y 7 pollos/jaula en verano $\left(206,28 \mathrm{~cm}^{2} / \mathrm{kg}\right)$.

Los pollos no deben ser sometidos a un período de ayuno mayor a doce horas entre la captura y la insensibilización en planta de faena, lo cual es importante para disminuir las posibilidades de contaminación microbiológica de las carcasas durante el proceso de eviscerado. El agua de bebida no se debe retirar hasta el comienzo de la carga ${ }^{25}$

\section{TRANSPORTE DE AVES}

El conductor del transporte debe conducir con precaución, procurando evitar maniobras que produzcan que los animales se caigan, sean aplastados y sufran traumas que eleven el nivel de estrés ${ }^{30}$. El tiempo de viaje no debe superar $6 \mathrm{~h}$ y el período de ayuno $12 \mathrm{~h}$ hasta el momento del sacrificio de las aves ${ }^{26}$. El tiempo de espera en planta de faena varía según la operatoria y la distancia del viaje (debería ser entre 30 minutos y 3 horas). Es importante respetar este tiempo mínimo para que los animales reduzcan el nivel de estrés que se manifiesta por disminución de frecuencia cardíaca y vocalizaciones ${ }^{24}$. 


\section{PLANTA DE FAENA}

La faena es la etapa donde culmina la cadena de producción y en la cual se evalúan los efectos de la aplicación del BA en todas las etapas previas. La faena debe realizarse de acuerdo a SENASA ${ }^{21,23}$ y a reglamentos internacionales ${ }^{4,5,12}$, relativos a la protección de los animales al momento de la matanza, siendo exigida y auditada anualmente por los mercados europeos para la exportación de productos argentinos.

Antes de que el camión ingrese a la planta de faena, las aves debieron ser pesadas en la balanza ${ }^{30}$. Ellas se trasladan a la zona de descanso, la cual debe disponer de paredes y techo que ofrezcan protección contra las inclemencias climáticas, que incluyan en lo posible poliuretano expandido u otro material para aislar la zona.

La entrada y salida deben ser abiertas para permitir la circulación de aire, con iluminación tenue a fin de calmar las aves. Además, debe contar con un sistema de forzadores de aire en más de una dirección (viento cruzado) a fin de generar un movimiento y renovación de aire confortable para las aves. También son importantes para las épocas estivales los aspersores de agua en forma de spray o foggins, que ayudan a refrescar y disminuir la sensación térmica ${ }^{25}$.

Estos sistemas deben estar regulados automáticamente para mantener la temperatura de termo-neutralidad en pollos parrilleros $\left(18-21^{\circ} \mathrm{C}\right)^{13}$. En planta de faena, el responsable de BA debe asegurarse de que todos estos parámetros se cumplan y debe verificar el estado de salud de las aves, la presencia de signos de lesiones y/o de estrés por calor o frío, dependiendo de la época del año. Los signos de estrés por calor se identifican por jadeo leve a intenso, alas abiertas y vocalización. Los signos de estrés por frío incluyen amontonamiento dentro de la jaula y alas pegadas al cuerpo ${ }^{13}$.

En las empresas argentinas de mayor envergadura, se aplican las especificaciones que se describen a continuación ${ }^{30}$. Los camiones para transporte de aves deben poseer techo y parte frontal cubierto con lona blanca en toda su superficie, en buen estado, a fin de proteger las aves del sol y la lluvia. También deben disponer de una reja por debajo del techo, para evitar que se escapen las aves del piso superior en caso de que se abra alguna tapa de las jaulas.

Este sistema puede ser manual, con poleas o hidráulico. En los laterales se debe contar con sujetadores, los cuales son listones metálicos que se acoplan en piso y techo para mantener las jaulas juntas y firmes. También es importante la utilización de separadores de madera que permitan la circulación de aire entre las aves, para lo cual se puede utilizar un listón a lo largo del chasis que separa las filas en dos grupos (izquierdo $\mathrm{y}$ derecho). El piso del chasis tiene que estar íntegro y debe facilitar el movimiento de las jaulas por arrastre en el momento de la descarga, a fin de evitar accidentes.

Luego del período de espera en zona de descanso, el transporte se dirige a la zona de descarga de jaulas. Es aquí donde las aves toman los primeros contactos con el personal de planta. La descarga de las aves puede ser manual o automática. En el primer caso, dos personas bajan cada fila de jaulas arrastrando la misma despacio y con cuidado, hasta la cinta transportadora.

Estos movimientos deben ser suaves a fin de evitar golpes, que pueden producir hematomas en las aves, sobre todo en la pechuga. Este mismo sistema puede ser automatizado mediante la utilización de un robot hidráulico que envía, por grupos, jaulas a la cinta transportadora. El equipo permite regular la configuración de velocidad y fuerza, a fin de hacerlo lo más suave posible.

La evaluación de BA en plantas de faena incluye dos indicadores, las aves muertas al arribo (AMA) y las lesiones en carcasa. El porcentaje de AMA permite conocer si las maniobras de carga y transporte a planta de faena fueron las adecuadas ${ }^{7}$; un operario entrenado retira las aves que llegan muertas y las coloca en recipientes de decomiso; registrando los datos de modo digital y manual, para su posterior control por el encargado de BA de SENASA. El porcentaje máximo aceptable de AMA es $0,5 \%$ del total de la faena del día ${ }^{30}$.

Una vez que las jaulas están en la cinta transportadora, se dirigen a la "zona de colgado", que es un área físicamente separada de la descarga. Este lugar debe poseer suficiente cantidad de forzadores de aire, y la particularidad es la iluminación con luz azul muy tenue (longitud de onda $340-380 \mathrm{~nm}$ ), valores de longitud de onda que no son percibidos por las aves y permiten mantenerlas tranquilas ${ }^{26}$.

En esta etapa, el encargado de BA debe supervisar la técnica correcta de colgado, la cual consiste en tomar al ave de un muslo, levantarlo e inmediatamente tomar el otro muslo con la otra mano sacando el ave de la jaula. Luego se la levanta cuidadosamente y se cuelga de la percha metálica con suavidad, a fin de evitar estrés por miedo y golpes en los muslos. Seguidamente, un operario debe verificar al final de la cinta que no haya quedado ningún ave en las jaulas, ya que éstas ingresan en forma inmediatamente posterior a la máquina lavadora con agua a presión y solución desinfectante ${ }^{23,30}$.

El tiempo transcurrido entre el colgado del ave y su insensibilización no debe superar los 45 segundos ${ }^{30}$, a fin de prolongar el menor tiempo posible la posición vertical invertida, que es sumamente estresante para las aves, ya que no poseen diafragma; por lo cual pueden experimentar sofocamiento y asfixia. Seguidamente las aves suspendidas en los ganchos llegan al área de insensibilizado, separada físicamente del colgado. Este procedimiento es denominado técnicamente electronarcosis por inmersión en baño de agua múltiple.

Las aves colgadas por las patas están conectadas al circuito, por un lado por las perchas que las sostienen y por otro lado toman contacto con el agua electrificada (sumergidas hasta la base de las alas, por un período no menor a 4 segundos) ${ }^{23}$.

De esta manera se cumple la ley de $\mathrm{Ohm}$, donde la intensidad de corriente que circula por el circuito (amperios), es la suma de las intensidades de corriente que 
pasa por cada una de las aves y esta última depende de la diferencia de potencial aplicada en el circuito (voltios) y la propia resistencia (ohmios) del ave (a mayor tamaño del ave, mayor resistencia ofrecerá al paso de la corriente) ${ }^{2}$.

Es muy importante que el encargado de BA del establecimiento evalúe la eficacia de la insensibilización en una muestra representativa, en general a la velocidad que trabajan las plantas de nuestro país, que requieren un control de 30-45 segundos ${ }^{17,30}$. Los signos de un correcto insensibilizado son: cuello arqueado, alas pegadas al cuerpo, mirada perdida, pupilas dilatadas, ausencia de reflejo palpebral, ausencia de aleteo, ausencia de reacción frente a estímulos del entorno y cese de la respiración ${ }^{13}$.

Un animal se considera inconciente cuando no despierta y no presenta signos de respuestas positivas o negativas como el miedo o la excitación; y puede considerarse insensible cuando carece de reacciones ante estímulos como el sonido, el olor, la luz o el contacto físico ${ }^{6}$. Si en este momento se realizara un electroencefalograma, mostraría un patrón de ondas de tipo epiléptico, seguido de un patrón de supresión profunda de actividad de la corteza cerebral o patrón isoeléctrico, señales consideradas por los investigadores como inequívocas de inconciencia e insensibilidad ${ }^{4,14}$.

El equipo de insensibilizado debe ser sometido a calibraciones periódicas por personal técnico especializado y documentar las mismas. También es importante contar con un equipo de aturdido extra en caso de que ocurra algún desperfecto, para asegurar que la faena continúe con normalidad ${ }^{20}$.

Luego de la insensibilización se procede al degüello. El tiempo máximo entre el insensibilizado y el degüello no debe superar los 10 segundos; de esta manera se puede asegurar que las aves no recuperen la conciencia. El degüello se realiza con cuchilla circular automática en la base del cuello; la profundidad debe ser tal que asegure la sección de los grandes vasos (arterias carótidas y venas yugulares) a fin de obtener un correcto sangrado y una muerte rápida ${ }^{25}$.

La muerte de las aves se produce por efecto de la isquemia cerebral. Actualmente, se considera que el tiempo medio necesario para producir la muerte con un corte de dos carótidas es de 15 a 20 segundos aproximadamente ${ }^{17}$.

El equipo de degüello debe estar correctamente calibrado y las cuchillas se cambian una vez en cada turno de faena. Asímismo, el personal responsable debe ser capaz de reconocer si el corte fue correcto y si los signos indican la muerte. Para reforzar el degüello debe haber un operario que repase las aves con cuchillo manual, solo a aquellas aves que no pasaron por la cuchilla (por ejemplo, porque el cuello se salió de la guía) ${ }^{25} 30$. Esta actividad no debe superar las 30 aves en 3 minutos; de lo contrario se deben tomar medidas correctivas para calibrar el equipo ${ }^{30}$.

A continuación prosigue el sangrado, que debe implementarse en 30-60 segundos en promedio para todo el proceso ${ }^{25}$, a fin de garantizar que sea rápido y completo. Es importante que todas la aves se encuentren sin vida para continuar con el paso siguiente de la faena: el escaldado, en el cual las aves serán sumergidas en agua caliente a una temperatura de $52^{\circ}$ a $56^{\circ} \mathrm{C}$, durante aproximadamente 3 minutos, para ablandar los folículos de las plumas.

La siguiente etapa es el desplume y -seguidamente- se debe evaluar la integridad de la piel del ave, poniendo especial énfasis en la detección de callos en la pechuga (dermatitis pectoral), causados por contacto con la cama húmeda y las deyecciones. La presencia de lesiones en la carcasa son observadas, de acuerdo a las planillas de procedimientos operativos estandarizados, luego del desplume y posterior trozado. Se debe identificar la presencia de fracturas o dislocaciones en alas, articulaciones coxofemorales y hematomas en carcasa, entre otras ${ }^{18,21,23,26}$.

A continuación, se evalúa en una muestra representativa $(0,1$ al $1 \%)$, la presencia de lesiones en las garras, que son indicadoras importantes de BA. Las garras son muy apreciadas en los mercados asiáticos, representando su exportación, importantes ingresos ${ }^{8}$. El grado de lesiones plantares se clasifican en cuatro categorías (A, B, C y descarte). La sumatoria entre garras tipo C y descarte no debe superar el $10 \%$ de la muestra. Los resultados de clasificación de garras deben ser informados al personal que se desempeña en granjas de engorde, a fin trabajar en conjunto para mejorar estos indicadores ${ }^{21}$.

El siguiente sector de la faena es el trozado, en el cual se observan las características de carcasas y piezas, identificando presencia de fracturas, dislocaciones y hematomas. Estas evaluaciones las realiza el veterinario acreditado ante SENASA ${ }^{21,27}$. De este modo, es posible identificar el momento en que las lesiones fueron ocasionadas. Las dislocaciones sin sangre e/o inflamación no fueron producidas en el animal vivo, sino luego de la faena, secuela de la mecánica de la mis$\mathrm{ma}^{15,21}$. Por otro lado, si se observan hematomas rojos, podrá inferirse que ocurrió un golpe en los momentos previos a la faena, o incluso durante el transporte

Si la lesión observada es de color púrpura, podrá inferirse que fue producida durante la carga y recolección en granja de engorde, y si es de color verde se produjo días previos a la carga, ya que los macrófagos dérmicos han metabolizado la hemoglobina de la sangre acumulada en el trauma y generan este color por acumulo de bilirrubina. Por último, si la lesión es reducida y de una coloración naranja/amarillenta, es porque se produjo aún antes que la anterior, también en granja de engorde ${ }^{15}$.

\section{BIENESTAR ANIMAL}

En Argentina, los aspectos legales del bienestar animal están legislados por la ley $14.346 / 54$ de protección contra el maltrato y crueldad animal. Además, el SENASA estableció normas para regular el BA en to- 
das las acciones que implican la producción y faena de aves ${ }^{21,26}$.

\section{CONCLUSIONES}

Por motivos éticos y legales, el bienestar animal debe ser contemplado en cada etapa de la vida y faena de los animales de producción. Por estos intereses, no es sorprendente el continuo avance científico en estos temas que dan base a las normativas.

En Argentina, los procedimientos han sido estandarizados por normas nacionales e internacionales y su aplicación aporta a la calidad de vida y a la faena libre de sufrimiento. Ambos aspectos posibilitan que los consumidores accedan a alimentos de origen animal, en los cuales el bienestar está garantizado.

Agradecimiento: Al Dr. Claudio Machado, por los aportes realizados y la revisión del presente artículo.

\section{REFERENCIAS}

1. Bautista Y et al. 2016. Efecto del estrés por calor y tiempo de espera ante mortem en las características fisicoquímicas y la calidad de la carne de pollo. Arch Med Vet 48: 8997.

2. Bilgili SF. 1992. Electrical stunning of broilers. Basic concepts and carcass quality implications: a review. $J \mathrm{Appl}$ Poultry Res 1: 35-46.

3. Berg C, Raj AB. 2014. Procesado de carnes. Métodos de aturdido para aves: aspectos de bienestar animal. Proc XIVth European Poultry Conference, Stavanger, Norway, p. 142-152.

4. Comunidad Europea. 2007. Diario Oficial de la Unión Europea. Directiva 2007/43/CE. https:/www.boe.es/ doue/2007/182/L00019-00028.pdf .

5. Comunidad Europea. 2009. Reglamento CE 1099/2009 relativo a la protección de los animales al momento de la matanza. https://eur-lex. europa.eu/LexUriServ/Lex UriServ.do? uri=OJ:L:2009:303:0001:0030:ES:PDF

6. Filan SL, Llewellyn RH. 2006. Animal-assisted therapy for dementia: a review of the literatura. Intern Psychogeriatr 18: 597-611.

7. Grandin T. 2009. Poultry slaughter plant and farm audit: critical control points for bird welfare. https:/www.grandin.com/welfare.audit.using.haccp.html.

8. Li N, Ren Z1, Li D, Zeng L. 2020. Review: automated techniques for monitoring the behaviour and welfare of broilers and laying hens: towards the goal of precision livestock farming. Animal. Mar 14: 617-625.

9. Ministerio de Agricultura, Ganadería y Pesca. 2019. Información Estadistica: carne aviar. https:/www. magyp.gob.ar/sitio/areas/aves/estadistica/carne/_archivos $/ / 000001$ Indicadores $\% 20$ Mensuales $/ 000005$ Exportaciones.pdf.

10. Nicol CJ, Davies A. 2010. Bienestar de las aves de corral en los países en desarrollo. http:/ www.fao.org/3/al720s/ al720s00.pdf.
11. Organización Mundial del Comercio. 2015. Entender la $O M C, 5$ ta. Edición, Ginebra, Suiza, p. 116.

12. Organización Mundial de Sanidad Animal. 2017. Estrategia mundial de bienestar animal de la OIE. https://www. oie.int.

13. Organización Mundial de Sanidad Animal. 2019. Bienestar animal y sistemas de producción de pollos de engorde. Código sanitario para los animales terrestres de la OIE, Capítulo 7.10.

14. Plano, M. 1995. Aves comerciales y su medio ambiente, Edit. Pegaso, Buenos Aires, República Argentina, p. 7983.

15. Ricaurte SL. 2005. Problemas del pollo de engorde antes y después del beneficio. Rev Electrón de Vet (RedVet), VI: 6.

16. Redalyc Org. 2019. Recuentos microbiológicos de canales de aves. Efecto del tiempo de ayuno. Red. Vet. R-ISSN 1695-7504, https://www.redalyc.org/articulo.oa.

17. Robins A, Phillips CJ. 2011. International approaches to the welfare of meat chickens. World's Poultry Science Journal 67: 351-369.

18. Rowe E, Dawkins MS, Gebhardt SG. 2019. A systematic review of precision livestock farming in the poultry sector: is technology focussed on improving bird welfare? https:// www.ncbi.nlm.nih.gov/pmc/articles/PMC6770384.

19. Sabow AB et al. 2017. High frequency pre-slaughter electrical stunning in ruminants and poultry for halal meat production: A review. https://doi.org/10.1016 j.livsci.2017.05.021

20. Sanz, M. 2013. Aturdido eléctrico en baño de agua, $50^{\circ}$ Congr. Científ. de Avicultura, Asoc. Mundial Avicult. Científ. (WPSA) y Asoc. Españ. de Avicult (AECA). https://www.wpsa-aeca.es/aeca_imgs_docs/manuel_sanz. pdf

21. Servicio Nacional de Sanidad Animal (SENASA). 2009. Pautas a tener en cuenta en la inspección ante y post mortem en aves y lagomorfos. http://www.senasa.gob. ar/prensa/intranet/DNICA/DIPOA.pdf

22. Servicio Nacional de Sanidad Animal (SENASA). 2013. Manual de Buenas Prácticas de Bienestar Animal en el Transporte Terrestre de Animales. http://www.ciap.org.ar/ Sitio/Archivos/Manual.pdf

23. Servicio Nacional de Sanidad Animal (SENASA). 2014. Manual de buenas prácticas de bienestar animal en la faena de aves y lagomorfos. https:/www.argentina.gob.ar/ sites/default/files/bienestar_plantas_de_faena.pdf

24. Servicio Nacional de Sanidad Animal (SENASA). 2014 Resolución $N^{0} 46$. http://www.senasa.gob.ar/normativas/ resolucion-46-2014-ministerio-agroindustria

25. Servicio Nacional de Sanidad Animal (SENASA). 2015a. Bienestar animal. Un enfoque práctico para el buen manejo de especies domésticas, durante su tenencia, producción, concentración, transporte y faena. Versión 1 , pp 164. http://www.senasa.gob.ar/sites/default/files/bienestar_animal.pdf

26. Servicio Nacional de Sanidad Animal (SENASA). 2015b. Reglamento de productos subproductos y derivados de origen animal. Decreto 4238/68. http://www.senasa. gob.ar/decreto- 423868 
27. Servicio Nacional de Sanidad Animal (SENASA). 2018a. Resolución 575/2018. https:/www.senasa.gob.ar/ sites/default/files/arbol_senasa/informacion/normativa/ resol_y_anexos $/ 2018 / \mathrm{r}$ _senasa_575-2018 p.

28. Servicio Nacional de Sanidad Animal (SENASA). 2018b. Manual de bienestar animal para sistemas de producción de pollos de engorde. Anexo: http://servicios.infoleg. gob.ar/infolegInternet/anexos / 310000-314999/314358/ res575.pdf

29. Vieira RH, Dabdab WP, Soster CP, Tomas NT. 2017. How are information technologies addressing broiler wel- fare? A systematic review based on the welfare quality ${ }^{\circledR}$ assessment. https:/www.mdpi.com/2071-1050/12/4/1413

30. Virués OJ, Buela CG. 2006. Psychophysiological effects of human-animal interaction: Theoretical issues and long term interaction effects. J Nerv \& Mental Dis 194: 52-57.

31. Wigham E, Grist A, Mullan S, Wotton S., Butterworth A. 2019. The influence of welfare training on bird welfare and carcass quality in two commercial poultry primary processing plants. https://www. mdpi.com/2076$2615 / 9 / 8 / 584$ 